\title{
The Application of Lens Language in Animation Film Design
}

\author{
Jinan $\mathrm{Du}^{1}$, Zixiao Gong ${ }^{2 *}$ \\ ${ }^{1}$ Chiang Mai University, No. 239, Huiqiao Road, Sudeci District, Chiang Mai Thailand \\ ${ }^{2}$ London College of Communication, University of the Arts London, Elephant\&Castle, London SE1 6SB, England \\ ${ }^{*}$ Corresponding author. Email: Qqppdujinan@outlook.com
}

\begin{abstract}
Film and television language is a kind of artistic language. It directly attracts the audience's audio-visual senses, with an intuitive, specific, bright image to convey meaning, has a strong artistic appeal. The montage produced by camera movement and different camera clips formed the rules of the composition of screen images. It improves the grammar and rhetoric rules of film and television language. Animation is a dynamic picture language, and it has its text, syntax, language changes and syntax. The addition of film and television lens language can enrich the visual effects of animation. With the development of technology, their union is becoming closer and closer.
\end{abstract}

Keywords: Camera language, The lens, Animation, The movie

\section{INTRODUCTION}

An excellent film and television work so that the audience can receive understandable information; each lens of good or bad determines the overall effect of the final formation. An animated film is like a building. To build a magnificent and striking building, it must have high-quality raw materials. A pile of broken pieces is impossible to build. This is a guarantee of quality, so the lens language is equivalent to construction. The necessary raw materials, the film uses the language of the lens to convey its inner meaning. The film producer uses various means and methods to edit and combine multiple shots to express the film language with a specific meaning and tell the audience a complete story. Any language has established rules and symbols. These symbols are combined into several rules. These rules are equivalent to the language skills of using animated shots. Therefore, this article starts from the essential characteristics of the lens language and has a deep understanding. Promote the practical application of lens language in the production of animated films.

\section{THE CONCEPT OF LENS LANGUAGE}

Peoples always talk about movies, TV dramas, animations, or other film and television works in daily lives. People often hear words such as "picture" and "plot". "picture" is one shot after another, and "plot" is composed of "picture", which is another appellation and embodiment of lens language. A lens is a short continuous picture taken by a camera at a fixed or unfixed location. Lens language embodies the producer's inner feelings and thoughts expressed by the combination of shots, which is reflected in the combination of shots and reflected in a single shot.

\section{CLASSIFICATION AND APPLICATION SKILLS OF CAMERA LANGUAGE}

Generally speaking, camera language can be divided into two main categories: moving shots and fixed shots. Fixed lens means that the camera instrument position remains unchanged, the lens focal length remains unchanged, and the picture taken is the "fixed lens". Compared with a fixed lens, the camera instrument's position and the lens's focal length will change. The lens of the sport can also be divided into push Lens, zoom, pan, follow, move, comprehensive sports lens, which is what we often say "push, pull, shake, move, use". According to the different shooting angles, it can also be divided into head-up shots and top-down shots. Lens and head-up lens: The lens's focal length can be divided into the standard lens, short focal length lens, long focal length lens, and zoom lens. According to the different observation angles, it can be divided into objective, subjective, and positive and negative lenses. The combination of these shots makes the lens language of 
film and television works have rich changes and abundant emotional thoughts, so that film and television work maintain the continuity of the plot and mobilize the emotions of the audience ${ }^{[1]}$.

The use of shot language in animated films is as complex as that in live-action films. This section analyzes fixed shots and moving shots.

\subsection{Application skills of fixed lens}

In previous animated films, there was always an empty shot to tell the audience the location, environment, or characters of the story to help the audience get into the viewing state better. The use of empty shots is the use of language skills of fixed shots by animators. In order to give full play to the expressive force of empty shots in films, animators need to master the skills to satisfy the aesthetic taste of empty shots in the composition of visual effects. For example, in the animated film "The King and the Bird", the animator chose to use an empty shot to explain the place where the story happened, which fully demonstrated the magnificence of the Kingdom of Takkati[2]. The animator uses a simple empty lens composition to design the scene and the kingdom's image and uses a fixed lens language to create a sense of solemnity and impact for the audience.

\subsection{Application skills of motion shots}

Pushing lens, pulling lens, shaking lens, moving lens, and following lens are the most common five lenses.

\subsubsection{Push the lens}

Pushing the lens means that the lens gradually advances toward the picture-the scene becomes smaller - the subject becomes larger - the picture that the audience sees changes from far to near-to a partial panorama. Pushing shots can highlight the details of the subject, thereby emphasizing key images and important plots and forming additional emotional guidance and psychological hints to the audience. In "The Lion King", Scar takes Simba into a valley, where coyotes drive animals from the mountain to the foot of the mountain. In this set of classic passages, the design of the lens played a significant role. For example, when Simba saw animals rushing down from the valley, a bullet shot towards Simba, giving him a strong visual impact. People's mood also becomes tense.

\subsubsection{Pull the lens}

Pulling lens is a shooting method in which the camera gradually moves away from the subject or changes the lens's focal length so that the frame is separated from the subject from the near distance. Pulling the camera pulls the background space to the distance, which makes the audience have a psychological reaction of distance and shows the position of the main characters or scenery in the environment. For example, in the movie "Prince of Egypt", there are dynamic shots of Moses: facial features, Moses' body, a smoky panorama when Moses is smoking, etc. It reflects the part and the whole in the same shot for the audience and plays the effect of rendering the scene's atmosphere, highlighting the smoothness of the action.

\subsubsection{Pan the lens}

Camera panning refers to the camera's fixed position, which shoots objects by turning the lens left and right or up and down and guides the audience's eyes to sweep from one end of the picture to the other. The camera shake shows the content coherently so it requires the beginning and the end of the shot to have a clear purpose, from a specific target to be shot, and a series of processes between the two shots should also be the content to be shown. Since there are shooting objectives from the beginning to the end of the camera, it is necessary to consider the composition of the picture and ensure that the whole process of the camera is beautiful, complete, and harmonious.

\subsubsection{Remove the lens}

Camera shift means that the camera stays in the same position by moving the background up, down, left, and right. When moving, the camera is always in the moving picture so that the central position of the moving frame and the visual displacement have a sense of the scene. The space of the audience moving the lens is complete and coherent. Generally speaking, all moving camera animation paper is more than twice as long as ordinary paper. So moving camera animation paper can better reflect the three-dimensional sense of translation.

\subsubsection{Follow the lens}

Follow the lens, also known as follow the shot, refers to the camera always following the motion of the subject movement together with the shooting. The camera quickly shows the structural relationship between the complex architectural space and the environmental space, showing the dynamic subjective sight, causing the audience to feel personally on the scene. For example, in the animation "Tarzan", the first ending animation has a classic follow-up shot. Tarzan glided among the vines to lead to a beautiful, lush jungle to explain the ending. A complex follow-up shot with a flying eagle as the subject in The Pony King. The eagle at this time guides the viewer's sight, affecting the viewer's mood so that the viewer with an anxious state of mind waiting for the suspense of relief. In Forrest 
Gump, the feather in the opening credits also uses a similar follow-up shot, which also uses a feather to bring the audience into the story and lead the protagonist $^{[3]}$.

\section{ADVANTAGES AND APPLICATION OF LENS LANGUAGE IN ANIMATED FILMS}

The essence of animation film and television art is to reflect the thoughts, feelings, and thoughts that animation works want to express. In creating animation works, artistic expression and story rhythm should be reflected, and the role should be full of vigor and vitality.

Unlike movies, the lens of a movie needs to be determined by the movement and placement of the camera position, and the scheduling of scene objects and characters is relatively convenient. In the two animated films and movies, the picture of a lens is embodied by the shooting design, without particular scene objects. However, it can be embodied by painting on paper. It is not the authenticity and sense of space of the lens. Correspondingly, although there is no need to prepare the props needed for the movie to reflect the lens's three-dimensionality and stability, the animation scene design must be carefully considered. Each object on the paper has its characteristics. There are also higher requirements for rationality, space, and perspective, which have very high requirements for the plotter design of the line splitter. Cartographers need to simulate the fundamental shooting skills, photography scheduling and action of the film. On this basis, the screen design can increase exaggeration so that the animation and reality can be more clearly distinguished. The lens in animated film and television needs to produce the motion effect required by the photographic equipment from the picture changes, which needs to take the lens language as the carrier to make the picture get the most immediate and vivid sense of the lens.

The second advantage is that the shot language of animated films and television can achieve a shot language that is impossible or difficult to achieve in films and at a lower cost. Movies or other film and television productions require virtual scenes shot on site or simulated by modern science and technology. The former requires enormous investment, while the latter requires high technology. Animation can do this more easily. It can even be changed according to the scene reference image to draw the desired scene effect, such as shooting sci-fi and surreal scenes, and animation can be easily achieved. In the animation production process, each shot needs to be rendered and processed with rich details to express better lens language. This also requires a lot of time, money, and workforce, but compared with movies, and these costs are three factors Much less [4].
With the support of national policies, the animation film and television industry ushered in a new spring. Many excellent works emerge in an endless stream, but most are still of solid commercial nature. Although the grasp and use of lens language have technically reached a relatively mature stage, the lack of its artistry is a problem we need to notice. In order to give full play to the lens language in the creation of animated film and television works, we should make reasonable use of lens language and make good use of its advantages in the creation of animated film and television. As a branch of film and television works, animated films are basic to the natural and smooth connection of shots, and shot language is indispensable. The use of camera language lets the audience understand the film's main plot and wants to express their thoughts and feelings. In addition, the style and shot combination technique should also choose the same theme and content of the film, based on the law of film performance, give full play to creativity and artistry.

\section{THE CAMERA IS DESIGNED RELATIVE TO THE LENS}

As a virtual camera in lens design, the camera position is virtual, but the virtual position is subtle, which is related to the origin of animation. Animation has a very close relationship with other art form-comics. In the past, the appearance of comics was just simple pictures and blunt dialogues. Later, comics gradually added film techniques and film modelling techniques: composition, positioning, mirroring, scenes, etc., which enriched the comics. Such modern cartoons are called movie cartoons. Due to the popularity of movie comics and their similarities with animated movies, many famous comics have been adapted into animated movies. For example, the Japanese animation "Allale" and "Crayon Shin-chan" all fall into this category. In the cartoon composition of Crayon Shin-chan, the lens position is relatively flat. In the film, the camera's position seems to be fixed in several places forever, close-up, and long-distance scenes are widely used in landscapes, and close-ups and panoramas are rarely used. This is a flat technique, which is determined by the subject matter and content. This style is particularly suitable for animated TV series because it reduces much work. For example, if the same machine is used repeatedly in the same scene, only one background is enough. This is just one of many animation styles. On the contrary, Hong Kong director Tsui Hark's production style is entirely different. The animation of "Xiao Qian" he produced is very rich in composition and positioning. Tsui hark always likes to use his great Angle, and the position becomes stylized, which plays a significant role in the narrative.

There is a technique in the movie called "multicamera shooting". What appears in the picture is a series 
of shots depicting the same thing from different angles simultaneously. Producers can do this in cartoons. For example, Gauguin was shot dead in Pocahontas. Gauguin and Pocahontas face each other. The arrangement is as follows:

i. Close-up, a close-up of Gauguin's face when he was shot. (The position faces Gauguin);

ii. In the middle shot, Gauguin broke Pocahontas necklace with his hands when he fell backward. (The machine turns around and aims at Pocahontas);

iii. In the distant view, Gaogong retreats in the forest. (On the other side of the forest opposite Gauguin);

iv. Large close-up, head lowered. (The location is very close to Gauguin's head side);

v. Looking closer, Gauguin fell into the water. (The location is the subjective perspective of Pocahontas);

vi. CIC, Pocahontas is kneeling on the ground, and Gauguin is lying in the water. (Return to the audience).

This set of scenes is a typical movie language. The designer makes good use of multi-angle design, strengthens the authenticity of the scene, emphasizes Gauguin's sudden killing, and brings a new visual experience to the animated film. The location design in the animation should be diverse, and the style varies from movie to movie. The change of machine position can dissolve new vitality for animation [5].

\section{SHOT SCHEDULING IN LENS DESIGN}

In the American cartoon "The Lion King", there is a scene in which Scar deceives Simba into the valley, letting the little wolf from the mountain drive the animals to the bottom of the valley, and then used the Scar to inform Mufasa that Mufasa had intervened. The story is about the disaster of an animal running. In this group of classic models, the lens design also played a significant role. For example, when Simba sees an animal rushing down from the valley, he throws a shot at Simba, giving a strong visual impact. Another example: After Mufasa rescued Simba from being knocked down, Simba lay on the ground and waited for his father. The plane was arranged at a lower visual height. The audience and Simba experienced the shock of countless animal limbs passing by.

Therefore, a good camera design can add color to the story or even the film. Due to the limitation of animation shooting mode, the camera's motion is only translation, push and pull, which is very different from the actual shooting of film and television. In addition to pushing, pulling, shaking, and moving, there are also lifting shots, following shots, and swinging shots, which are challenging to achieve in two-dimensional animation shooting. The essence of animation in two-dimensional animation is that it is difficult to imitate elevators. Virtual characters vary significantly in two-dimensional Angle and are not easy to draw, mainly when the background rotates. Although animation can imitate all the shooting skills of movies, sometimes these imitation skills are laborious and thankless.

With the development of computers, some new art forms were born. It can be said that $3 \mathrm{~d}$ animation has suddenly risen and occupied an important position in animation production. This new thing has something that two-dimensional animation does not have, and it has unlimited potential advantages. Take the lens, for example, the difficulty of imitating elevators in twodimensional animation. Three-dimensional animation can be easily solved, much easier than actual camera shooting. In the world of 3D animation, by changing the camera movement and camera position settings, or as long as the photographer sets the camera movement tracking in the computer, the computer will display everything that has been designed. It can do whatever one wants in the three-dimensional world and bring people new visual experiences. The familiar "Frozen" and "Toy Story" are all 3d computer animation movies. Combining 3D and 2D images, 3D images, and real-life shooting creates an unprecedented space for animation and even blurs the boundaries between animation and film. For example, "Porky Little Mouse" is a combination of film and animation. For example, "Porky Little Mouse" is a combination of film and animation.

\section{CONCLUSION}

The animated film is a virtual world, and the lens design of the animation is also the lens design of the virtual reality camera. Except for the animated camera itself, all camera designs are drawn, moving to achieve the effect. Although the animation camera cannot produce some effects, such as rotating the lens and changing the angle of view, this is not a problem in the design of the animation lens. All fundamental shooting skills, photography auctions, and scene scheduling can be designed in animation. Animation shooting animation can imitate all basic shooting skills. The lens in animation is more expressive than the lens in real life because it lacks the constraints of the natural environment. The design of the animation camera picture includes the picture effect produced by the movement of the camera itself and the movement effect of the camera produced by the picture change. All these rely on film and television lens language to achieve a strong and true sense of the lens. 


\section{AUTHORS' CONTRIBUTIONS}

The author conducted the literature reviews and wrote this article. Zixiao Gong proofread this article based on his own teaching experience and put forward some suggestions. At the same time, the author would like to take this opportunity to thank the family and friends for their encouragement and support during the study.

\section{REFERENCES}

[1] Dai Xiaoling. The Application of film and television Shot language in animation [J]. Film Review, 2007(03):60+62.

[2] Wang Yi. On the lens language and its application skills in animated Films [J]. Fine Arts Education Research, 2013, 000(019):104-104.

[3] Han Rui. Silent Touch -- Analysis on the Performance and Application of animation Shot language [J]. Northern Literature (second half), 2012.

[4] Liu Siyang. Brief analysis on the application of shot language in animated short films [J]. Art Fashion: Theory Edition, 2014(5):53-53.

[5] Zhang Yun. Discussion on the application of shot language skills in animation films [J]. Journal of Anhui Electronic Information Vocational Technology College, 2014, 000(003):20-23. 